\title{
Passive scheme with photon-number-resolving detector for monitoring the untrusted source in Plug-and-Play quantum key distribution system
}

\author{
Bingjie $\mathrm{Xu}$, Xiang Peng. \\ CREAM Group, State Key Laboratory of Advanced Optical Communication \\ Systems and Networks (Peking University) and Institute of Quantum Electronics, \\ School of Electronics Engineering and Computer Science, Peking University, Beijing 100871, PR China
}

(Dated: October 30, 2018)

\begin{abstract}
A passive scheme with a beam splitter and a photon-number-resolving (PNR) detector is proposed to verify the photon statistics of an untrusted source in a plug-and-play quantum-key-distribution system by applying a three-intensity decoy-state protocol. The practical issues due to statistical fluctuation and detection noise are analyzed. The simulation results show that the scheme can work efficiently when the total number of optical pulses sent from Alice to Bob is above $10^{8}$, and the dark count rate of the PNR detector is below 0.5 counts/pulse, which is realizable with current techniques. Furthermore, we propose a practical realization of the PNR detector with a variable optical attenuator combined with a threshold detector.

PACS numbers: 03.67.Dd, 03.67.Hk
\end{abstract}

\section{INTRODUCTION}

Based on the BB84 protocol [1], the security analysis for the practical quantum-key-distribution (QKD) source was given [2, 3]. Further, the decoy-state protocol was proposed [4] and was developed [5-10] to improve the QKD performance. Commonly, a trusted QKD source is considered for those protocols, which means the photon-number distribution (PND) of the source is fixed and is known by Alice and Bob. However, this assumption is not always valid in practice. For example, the intensity fluctuation from the source and the parameter fluctuation from the optical devices cause the assumption of the trusted source to fail [11]. In particular, an untrusted source exists in a real-life experiment (i.e., twoway plug-and-play system) and gives rise to the possibility of a Trojan-horse attack [12-16], where the source is pessimistically controlled by Eve. Thus, the statistical characteristics of the QKD source need to be verified to boost the QKD performance [12-26].

Intuitively, if the characteristics of the untrusted source infinitely approaches that of the trusted source, Alice needs a quantum nondemolition (QND) measurement [27] to verify the PND of the QKD source. However, it is hard to implement the QND measurement in practice. Fortunately, an analytical method was provided to calculate the final key rate when the probability of untagged bits was known by Alice and Bob [13], and an active photon-number-analyzer (PNA) scheme was proposed to monitor the probability of untagged bits. However, it is challenging to implement the active scheme, where a high speed random optical switch and a perfect intensity monitor are needed. Then, using inverseBernoulli transformation, a passive scheme, with a beam splitter (BS) and an imperfect detector, was proposed and was verified experimentally [14]. Furthermore, to realize the passive scheme more efficiently, a high-speed two-threshold de-

\footnotetext{
*xiangpeng@pku.edu.cn.

†hongguo@pku.edu.cn.
}

tection can be used without applying inverse-Bernoulli transformation postprocessing [15, 16]. From another viewpoint, recent results for the three-intensity decoy-state protocol with the untrusted source have rigorously proved that it is sufficient to monitor the lower and upper bounds of the probability for Alice to send out vacuum, one photon, and two photon states [19, 20]. More recently, the detector-decoy scheme was theoretically proposed to monitor the PND of an untrusted source using a threshold detector combined with a variable optical attenuator (VOA) [22].

In the following, a passive scheme with a BS and a photon-number-resolving (PNR) detector, which can discriminate vacuum, one-photon, two-photon, and more than twophoton states, is proposed to monitor the parameters needed in Refs. [19, 20]. Then, the untrusted source in the plug-andPlay QKD system can be monitored with the passive scheme. Additionally, some practical issues due to finite-data size and random-detection noise are included in the analysis. Furthermore, a proposed realization of the PNR detector is analyzed based on the idea of the detector-decoy scheme [22].

\section{KEY PARAMETERS IN SECURITY ANALYSIS}

Generally, the secure key rate of the BB84 protocol is [2, 3]

$$
R=\frac{1}{2} Q\left\{\Delta_{1}\left[1-H_{2}\left(e_{1}\right)\right]-H_{2}(E)\right\},
$$

where $Q$ and $E$ are, respectively, the count rate and the quantum bit error rate (QBER) measured in the QKD experiment, $\Delta_{1}\left(e_{1}\right)$ is the fraction of counts (QBER) due to the singlephoton state, and $H_{2}(x)=-x \log _{2}(x)-(1-x) \log _{2}(1-x)$ is the binary Shannon entropy. In the standard security analysis of the BB84 protocol, all the losses and errors are assumed from the single-photon state [3], which gives

$$
\Delta_{1}=\frac{Q-P_{\text {multi }}}{Q}, e_{1}=\frac{E}{\Delta_{1}},
$$

where $P_{\text {multi }}$ is the probability for Alice to send out multiphoton states. 
The decoy-state method offers a more effective way to estimate the lower (upper) bound of $\Delta_{1}\left(e_{1}\right)$ compared to Eq. (2) [4-10]. In the three-intensity decoy-state protocol [6, 9], Alice randomly sends three kinds of sources: vacuum, decoy, and signal sources, respectively. The quantum state of the decoy (signal) source is $\rho_{d}=\sum_{n=0}^{\infty} a_{n}|n\rangle\langle n|$ $\left(\rho_{s}=\sum_{n=0}^{\infty} a_{n}^{\prime}|n\rangle\langle n|\right)$. For a three-intensity decoy-state QKD system with an untrusted source, it was proved that [19, 20]

$$
\Delta_{1}^{s} \geq \frac{a_{1}^{\prime L}\left(a_{2}^{\prime L} Q_{d}-a_{2}^{U} Q_{s}-a_{2}^{\prime L} a_{0}^{U} Q_{0}+a_{2}^{U} a_{0}^{\prime L} Q_{0}\right)}{Q_{s}\left(a_{1}^{U} a_{2}^{\prime L}-a_{1}^{\prime L} a_{2}^{U}\right)},
$$

where $Q_{0}, Q_{d}$, or $Q_{s}$ is the count rate of vacuum, decoy, and signal sources, respectively, and $\Delta_{1}^{s}$ is the fraction of counts due to the single-photon state in the signal source. To calculate the lower bound of $\Delta_{1}^{s}$, one needs to estimate the parameters $\left\{a_{0}^{\prime L}, a_{0}^{U}, a_{1}^{\prime L}, a_{1}^{U}, a_{2}^{\prime L}, a_{2}^{U}\right\}$, where the superscript $L(U)$ means lower (upper) bound. The secure key rate of the signal source is

$$
R^{s}=\frac{1}{2} Q_{s}\left\{\Delta_{1}^{s}\left[1-H_{2}\left(e_{1}^{s}\right)\right]-H_{2}\left(E_{s}\right)\right\},
$$

where $E_{s}$ is the QBER from the signal source and $e_{1}^{s}=E_{s} / \Delta_{1}^{s}$. In the following, we present a passive scheme to estimate the parameters $\left\{a_{0}^{\prime L}, a_{0}^{U}, a_{1}^{\prime L}, a_{1}^{U}, a_{2}^{\prime L}, a_{2}^{U}\right\}$.

\section{THEORY OF ESTIMATION WITH PASSIVE SCHEME}

The passive scheme for estimating the parameters $\left\{a_{0}^{\prime L}, a_{0}^{U}\right.$, $\left.a_{1}^{\prime L}, a_{1}^{U}, a_{2}^{\prime L}, a_{2}^{U}\right\}$ is shown in Fig. 1, where a PNR detector that can discriminate the photon number of $n=0, n=1, n=2$, and $n \geq 3$ is used. For simplicity, one can calibrate the setup to satisfy

$$
\eta_{D}\left(1-\eta_{B S}\right)=\eta_{B S},
$$

where $\eta_{B S}$ is the transmittance of the BS and $\eta_{D}$ is the detection efficiency of the PNR detector. Under this condition, the $\mathrm{PND}$ at $\mathrm{P} 4$ is the same as that at $\mathrm{P} 3$, where $\mathrm{P} i$ means position $i(i=1,2,3,4)$ in Fig. 1.

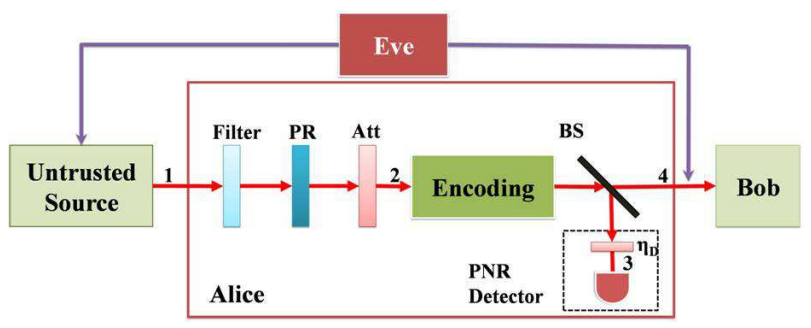

FIG. 1: (Color online) The untrusted source prepared at P1 by Eve, where $\mathrm{P} i$ means position $i(i=1,2,3,4)$, passes through an optical filter, a phase randomizer (PR), and an attenuator (Att) with the attenuation coefficient $\eta_{s}\left(\eta_{d}\right)$ for the signal (decoy) source. After the source is encoded, a BS with transmittance $\eta_{B S}$ separates it into two beams: One goes to a PNR detector with efficiency $\eta_{D}$ at P3, and the other is sent out from Alice's side at P4.

\section{A. PNR detector without detection noise}

In the following, the PNR detector is assumed to be noiseless. Suppose that $P^{s(d)}\left(n_{4}\right)$ denotes the PND for the signal (decoy) source at $\mathrm{P} 4$, and $D^{s(d)}(m)$ denote the PND for signal (decoy) source at P3. Clearly, one has

$$
\begin{aligned}
& a_{n}=P^{d}\left(n_{4}=n\right)=D^{d}(m=n), \\
& a_{n}^{\prime}=P^{s}\left(n_{4}=n\right)=D^{s}(m=n),
\end{aligned}
$$

where $n=0,1,2, \cdots$.

Suppose that the data size $N$ is the total number of optical pulses sent from Alice to Bob, while $N^{s(d)}$ is the number of signal (decoy) pulses, correspondingly. Let $k_{m}^{s(d)}$ denote the number of detected signal (decoy) pulses at P3 given that the PNR detector records $m$ photoelectrons $(m=0,1,2)$. Using the random sampling theory [28], each $D^{s}(m) \in\left[k_{m}^{s} / N^{s}-\right.$ $\left.\varepsilon^{\prime}, k_{m}^{s} / N^{s}+\varepsilon^{\prime}\right]$ with a confidence level $1-2 \exp \left(-N^{s} \varepsilon^{\prime 2} / 2\right)$ for signal pulses, and each $D^{d}(m) \in\left[k_{m}^{d} / N^{d}-\varepsilon, k_{m}^{d} / N^{d}+\varepsilon\right]$ with a confidence level $1-2 \exp \left(-N^{d} \varepsilon^{2} / 2\right)$ for decoy pulses can be estimated. For enough small $\exp \left(-N^{s} \varepsilon^{\prime 2} / 2\right)$ and $\exp \left(-N^{d} \varepsilon^{2} / 2\right)$, simultaneously, $D^{s}(m) \in\left[k_{m}^{s} / N^{s}-\varepsilon^{\prime}, k_{m}^{s} / N^{s}+\right.$ $\left.\varepsilon^{\prime}\right]$ and $D^{d}(m) \in\left[k_{m}^{d} / N^{d}-\varepsilon, k_{m}^{d} / N^{d}+\varepsilon\right]$ for $m=0,1,2$ are estimated approximately with a confidence level 1 -
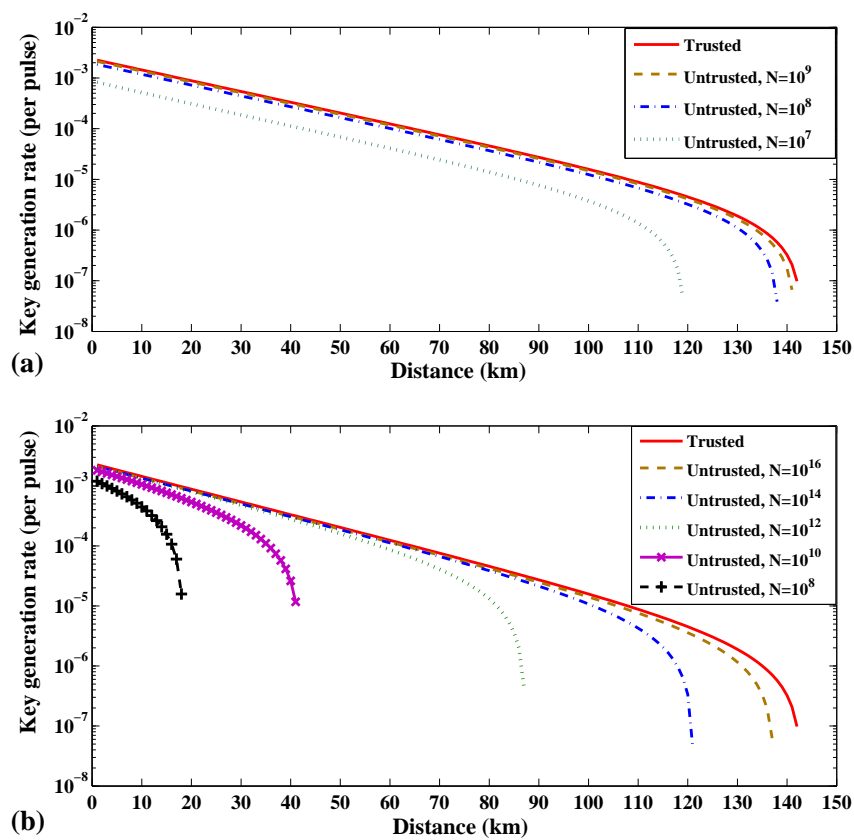

FIG. 2: (Color online) Simulation results of the three-intensity decoy-state protocol for the trusted source with infinite-data size, and for the untrusted source with: (a) finite-data size $N=10^{9}, 10^{8}, 10^{7}$, respectively, based on the passive PNR scheme in Fig. 1, where a BS and a noiseless PNR detector are used to verify the parameters $\left\{a_{0}^{\prime L}\right.$, $\left.a_{0}^{U}, a_{1}^{\prime L}, a_{1}^{U}, a_{2}^{\prime L}, a_{2}^{U}\right\}$ with a confidence level $1-10^{-6}$; (b) finitedata size $N=10^{16}, 10^{14}, 10^{12}, 10^{10}, 10^{9}$, respectively, based on the passive PNA scheme [15], where a BS and noiseless PNA are used to verify the lower bound of the probability of untagged bits with a confidence level $1-10^{-6}$ (see Appendix A). 
$6 \exp \left(-N^{s} \varepsilon^{\prime 2} / 2\right)-6 \exp \left(-N^{d} \varepsilon^{2} / 2\right)$. From Eq. (6), one simultaneously gets

$$
a_{m}^{\prime L}=\frac{k_{m}^{s}}{N^{s}}-\varepsilon^{\prime}, a_{m}^{U}=\frac{k_{m}^{d}}{N^{d}}+\varepsilon,(m=0,1,2)
$$

with a confidence level $1-6 \exp \left(-N^{s} \varepsilon^{\prime 2} / 2\right)-6 \exp \left(-N^{d} \varepsilon^{2} / 2\right)$.

For testing the effects of the finite-data size, we choose an untrusted source of Poissonian statistics to perform simulations based on the three-intensity decoy-state protocol. Figure 2(a) shows the numerical simulation results for the trusted source, and the untrusted source with the passive PNR scheme in Fig. 11, where a BS and a noiseless PNR detector are used to verify the parameters $\left\{a_{0}^{\prime L}, a_{0}^{U}, a_{1}^{\prime L}, a_{1}^{U}, a_{2}^{\prime L}, a_{2}^{U}\right\}$. Here, the average photon number (APN) of the Poissonian source at P1 is $7.69 \times 10^{6}$. The attenuation $\eta_{s(d)}$ is set to be $5 \times 10^{-7}\left(1 \times 10^{-7}\right)$, and the transmittance of the BS is $\eta_{B S}=0.13$ so that the APN for the signal (decoy) state at P4 is $\mu_{s}=0.5\left(\mu_{d}=0.1\right)$. The detection efficiency of the PNR detector $\eta_{D}$ is set to be 0.15, so that Eq. (5) holds. The photoelectron detection data recorded by the PNR detector is simulated using the Monte Carlo method, and $N=10^{7}, 10^{8}$, and $10^{9}$ of measurements are run. Other experimental parameters are cited from the GYS experiment [29] as shown in Table I] where $\eta_{B o b}$ is the efficiency of Bob's detection, $Y_{0}$ is the dark count rate of Bob's detector, and $e_{\text {det }}\left(e_{0}\right)$ is the probability that a photon (dark count) hits the erroneous detector on Bob's side. To compare the performance of the scheme in Fig. 1 with the passive PNA scheme proposed in Ref. [15], where a BS with transmittance $\eta_{B S}$ and a noiseless PNA with efficiency $\eta_{D}$ are used to verify the probability of untagged bits (see Appendix A), Fig. 2(b) shows the numerical simulation for the trusted source and the untrusted source with the passive PNA scheme. All the experimental parameters are chosen to be the same as that for Fig. 2(a).

\section{B. PNR detector with additive detection noise}

Given a PNR detector with an independent additive detection noise $y$, the detected photoelectron number $m^{\prime}$, and the photon number $m$ at $\mathrm{P} 3$ satisfy $m^{\prime}=m+y$. One can calculate the lower and upper bounds of PND $D(m)(m=0,1,2)$ at P3 based on the detected photoelectron distribution $P\left(m^{\prime}\right)$, given that the distribution of the detection noise $N(y)$ is known by Alice and independent of the input source. The dark count is the main kind of detection noise for the PNR detectors, such as the time multiplexing detector [30, 31], the transition-edge sensor [32], or a threshold detector together with a variable Att [22, 33]. In the case of independent Poissonian statistics noise, the probability of detecting $m^{\prime}$ photoelectrons is $P\left(m^{\prime}\right)=\sum_{d=0}^{m^{\prime}} N(d) D\left(m^{\prime}-d\right)$, where $N(y=d)=e^{-\lambda} \lambda^{d} / d$ ! is the probability that $d$ dark counts occur in the PNR detector, and $\lambda$ is the average dark count rate. Then, one has

$$
\left[\begin{array}{l}
D(m=0) \\
D(m=1) \\
D(m=2)
\end{array}\right]=\left[\begin{array}{ccc}
P\left(m^{\prime}=0\right) & 0 & 0 \\
P\left(m^{\prime}=1\right) & P\left(m^{\prime}=0\right) & 0 \\
P\left(m^{\prime}=2\right) & P\left(m^{\prime}=1\right) & P\left(m^{\prime}=0\right)
\end{array}\right]\left[\begin{array}{c}
e^{\lambda} \\
-e^{\lambda} \lambda \\
e^{\lambda} \lambda^{2} / 2
\end{array}\right] .
$$

Let $k_{m^{\prime}}^{S(d)}$ denote the number of detected signal (decoy) pulses by Alice at P3 given that the PNR detector records $m^{\prime}$ photoelectrons. Using the random-sampling theory [28], simultaneously, $P^{s}\left(m^{\prime}\right) \in\left[k_{m^{\prime}}^{s} / N^{s}-\varepsilon^{\prime}, k_{m^{\prime}}^{s} / N^{s}+\varepsilon^{\prime}\right]$ and $P^{d}\left(m^{\prime}\right) \in$ $\left[k_{m^{\prime}}^{d} / N^{d}-\varepsilon, k_{m^{\prime}}^{d} / N^{d}+\varepsilon\right]$ for $m^{\prime}=0,1,2$ are estimated with a confidence level $1-6 \exp \left(-N^{s} \varepsilon^{\prime 2} / 2\right)-6 \exp \left(-N^{d} \varepsilon^{2} / 2\right)$. Combining Eqs. (6) and (8), one yields

$$
\begin{aligned}
a_{0}^{\prime} \geq & e^{\lambda}\left(\frac{k_{m^{\prime}=0}^{s}}{N^{s}}-\varepsilon^{\prime}\right)=a_{0}^{\prime L}, \\
a_{1}^{\prime} \geq & -\lambda e^{\lambda}\left(\frac{k_{m^{\prime}=0}^{s}}{N^{s}}+\varepsilon^{\prime}\right)+e^{\lambda}\left(\frac{k_{m^{\prime}=1}^{s}}{N^{s}}-\varepsilon^{\prime}\right)=a_{1}^{\prime L}, \\
a_{2}^{\prime} \geq & \frac{\lambda^{2}}{2} e^{\lambda}\left(\frac{k_{m^{\prime}=0}^{s}}{N^{s}}-\varepsilon^{\prime}\right)-\lambda e^{\lambda}\left(\frac{k_{m^{\prime}=1}^{s}}{N^{s}}+\varepsilon^{\prime}\right) \\
& +e^{\lambda}\left(\frac{k_{m^{\prime}=2}^{s}}{N^{s}}-\varepsilon^{\prime}\right)=a_{2}^{\prime L}, \\
a_{0} \leq & e^{\lambda}\left(\frac{k_{m^{\prime}=0}^{d}}{N^{d}}+\varepsilon\right)=a_{0}^{U}, \\
a_{1} \leq & -\lambda e^{\lambda}\left(\frac{k_{m^{\prime}=0}^{d}}{N^{d}}-\varepsilon\right)+e^{\lambda}\left(\frac{k_{m^{\prime}=1}^{d}}{N^{d}}+\varepsilon\right)=a_{1}^{U}, \\
a_{2} \leq & \frac{\lambda^{2}}{2} e^{\lambda}\left(\frac{k_{m^{\prime}=0}^{d}}{N^{d}}+\varepsilon\right)-\lambda e^{\lambda}\left(\frac{k_{m^{\prime}=1}^{d}}{N^{d}}-\varepsilon\right) \\
& +e^{\lambda}\left(\frac{k_{m^{\prime}=2}^{d}}{N^{d}}+\varepsilon\right)=a_{2}^{U}
\end{aligned}
$$

with a confidence level 1-6 $\exp \left(-N^{s} \varepsilon^{\prime 2} / 2\right)-6 \exp \left(-N^{d} \varepsilon^{2} / 2\right)$.

TABLE I: The simulation parameters for Figs. 2, 3 and 6

\begin{tabular}{ccccccc}
\hline \hline$\eta_{D}$ & $\eta_{B S}$ & $\eta_{B o b}$ & $\alpha$ & $Y_{0}$ & $e_{\text {det }}$ & $e_{0}$ \\
\hline 0.15 & 0.13 & 0.045 & 0.21 & $1.7 \times 10^{-6}$ & $3.3 \%$ & 0.5 \\
\hline \hline
\end{tabular}

For testing the effects of detection noise, we choose an untrusted source of Poissonian statistics to perform simulations based on the three-intensity decoy-state protocol with the passive PNR scheme in Fig. 11 The untrusted source is of Poissonian statistics with APN $\mu=7.69 \times 10^{6}$ at P1, and the attenuations $\eta_{s}$ and $\eta_{d}$ are set to be $5 \times 10^{-7}$ and $1 \times 10^{-7}$, respectively. The other experimental parameters are cited from Table I. The photoelectron detection and additive Poissonian noise of the PNR detector are simulated using the Monte Carlo method, and $N=10^{8}$ and $10^{9}$ of measurements are run for Figs. 3 a) and 3 (b), respectively. Our analysis is not limited to the Poissonian noise case. Generally, when the random-positive detection noise $y$ with the probability $N(y)$ is known to Alice, one has

$$
\left[\begin{array}{l}
P\left(m^{\prime}=0\right) \\
P\left(m^{\prime}=1\right) \\
P\left(m^{\prime}=2\right)
\end{array}\right]=\left[\begin{array}{ccc}
D(m=0) & 0 & 0 \\
D(m=1) & D(m=0) & 0 \\
D(m=2) & D(m=1) & D(m=0)
\end{array}\right]\left[\begin{array}{l}
N(y=0) \\
N(y=1) \\
N(y=2)
\end{array}\right] .
$$


Thus, combining the results in Eqs. (6) and (10), one has

$$
\begin{aligned}
a_{0}^{\prime} \geq & \frac{k_{m^{\prime}=0}^{s} / N^{s}-\varepsilon^{\prime}}{N(y=0)}, \\
a_{1}^{\prime} \geq & \frac{\left(k_{m^{\prime}=1}^{s} / N^{s}-\varepsilon^{\prime}\right) N(y=0)-\left(k_{m^{\prime}=0}^{s} / N^{s}+\varepsilon^{\prime}\right) N(y=1)}{N^{2}(y=0)} \\
a_{2}^{\prime} \geq & \frac{k_{m^{\prime}=2}^{s} / N^{s}-\varepsilon^{\prime}}{N(y=0)}-\frac{k_{m^{\prime}=1}^{s} / N^{s}+\varepsilon^{\prime}}{N^{2}(y=0)} N(y=1) \\
& +\frac{k_{m^{\prime}=0}^{s} / N^{s}-\varepsilon^{\prime}}{N^{3}(y=0)} N^{2}(y=1)-\frac{k_{m^{\prime}=0}^{s} / N^{s}+\varepsilon^{\prime}}{N^{2}(y=0)} N(y=2) \\
a_{0} & \leq \frac{k_{m^{\prime}=0}^{d} / N^{d}+\varepsilon}{N(y=0)}, \\
a_{1} & \leq \frac{\left(k_{m^{\prime}=1}^{d} / N^{d}+\varepsilon\right) N(y=0)-\left(k_{m^{\prime}=0}^{d} / N^{d}-\varepsilon\right) N(y=1)}{N^{2}(y=0)} \\
a_{2} & \leq \frac{k_{m^{\prime}=2}^{d} / N^{d}+\varepsilon}{N(y=0)}-\frac{k_{m^{\prime}=1}^{d} / N^{d}-\varepsilon}{N^{2}(y=0)} N(y=1) \\
& +\frac{k_{m^{\prime}=0}^{d} / N^{d}+\varepsilon}{N^{3}(y=0)} N^{2}(y=1)-\frac{k_{m^{\prime}=0}^{d} / N^{d}-\varepsilon}{N^{2}(y=0)} N(y=2) . \quad(11)
\end{aligned}
$$

Therefore, once the distribution of the detection noise is known, the secure key rate can be estimated given the bounds of $\left\{a_{0}^{\prime}{ }^{L}, a_{0}^{U}, a_{1}^{\prime L}, a_{1}^{U}, a_{2}^{\prime L}, a_{2}^{U}\right\}$.

\section{A PROPOSED REALIZATION OF A PNR DETECTOR}

The PNR detector can be realized by a VOA (VOA) combined with a practical threshold detector as shown in Fig. 4(a) [22], which is equivalent to the model in Fig. 4(b). Suppose that the state of input source is $\rho_{\text {in }}=\sum_{n=0}^{\infty} p_{n}|n\rangle\langle n|$. In figure 4(b), after passing through an Att with efficient $\eta_{D}$, the state of the source becomes $\rho^{\prime}=\sum_{n=0}^{\infty} p_{n}^{\prime}|n\rangle\langle n|$, where $p_{n}^{\prime}=\sum_{m=n}^{\infty} p_{m}\left(\begin{array}{c}m \\ n\end{array}\right) \eta_{D}^{n}\left(1-\eta_{D}\right)^{m-n}$. When Eq. (5) holds, one has

$$
p_{n}^{\prime s}=a_{n}^{\prime}, p_{n}^{\prime d}=a_{n}
$$

Then, the source passes through the VOA with efficiency $\eta$, and the probability that the detector dose not click can be calculated as $p(\eta)=\sum_{n=0}^{\infty}(1-\eta)^{n} p_{n}^{\prime}[22]$. When we take the dark count of the threshold detector into account, it can be calculated as $p(\eta)=(1-\lambda) \sum_{n=0}^{\infty}(1-\eta)^{n} p_{n}^{\prime}$, where $\lambda$ is the dark count rate of the detector. If Alice varies the transmittance of the VOA $\eta \in\left\{\eta_{1}, \cdots, \eta_{M}\right\}$, she has a set of linear equations,

$$
p\left(\eta_{i}\right)=(1-\lambda) \sum_{n=0}^{\infty}\left(1-\eta_{i}\right)^{n} p_{n}^{\prime}, \quad(i=1, \cdots, M) .
$$

When she employs an infinite number of possible transmittance $\eta \in[0,1]$, she can always estimate any finite number of probabilities $p_{n}^{\prime}$ with arbitrary precision by solving Eqs. (13). However, it is not necessary for our purpose in which we are mainly concerned with the probability of vacuum, onephoton, and two-photon states, and, thus, only three different
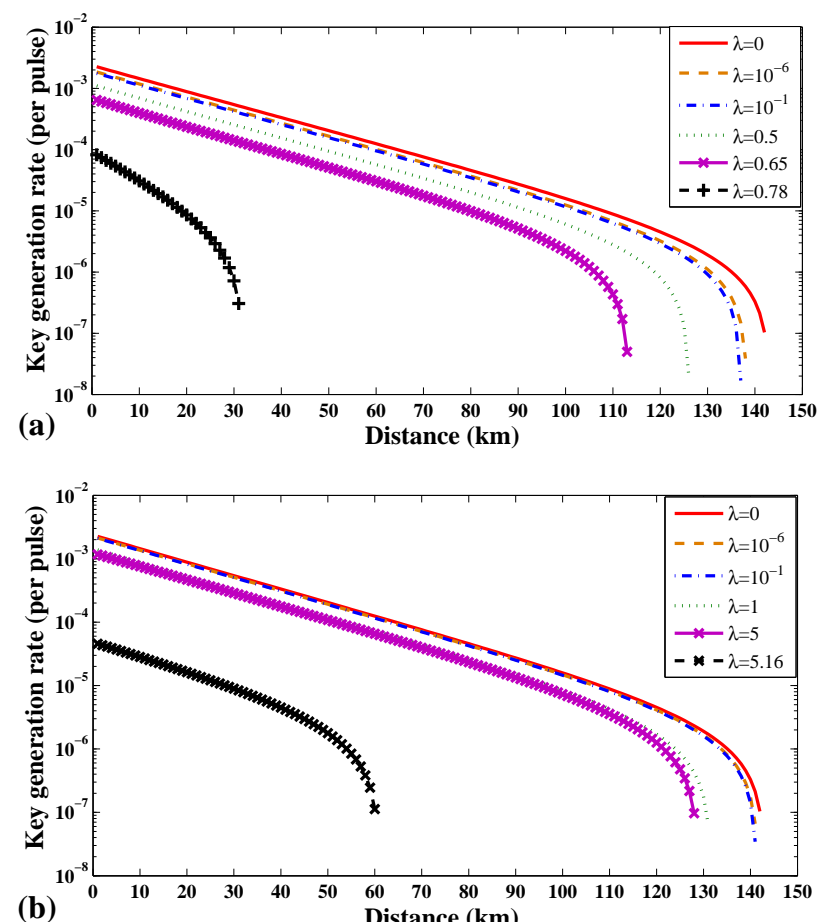

FIG. 3: (Color online) Simulation result of the three-intensity decoystate QKD with an untrusted source based on the scheme in Fig. 1: (a) The data size is $N=10^{8}$, and the APN of the Poissonian noise in the PNR detector is $\lambda=0,10^{-6}, 10^{-1}, 0.5,0.65,0.78$, respectively; (b) the data size is $N=10^{9}$, and the APN of the Poissonian noise in the PNR detector is $\lambda=0,10^{-6}, 10^{-1}, 1,5,5.16$, respectively. The experimental parameters are the same as in Table I. The confidence level for both cases is $1-10^{-6}$.

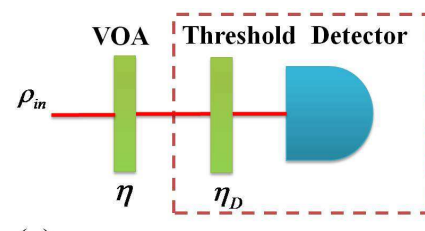

(a)

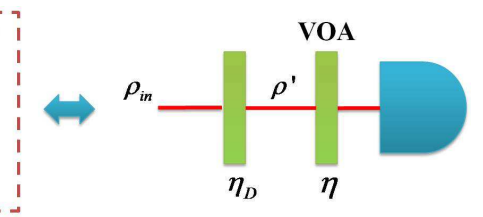

(b)
FIG. 4: (Color online) (a) A threshold detector (modeled by an Att with transmittance $\eta_{D}$ and an ideal threshold detector) combined with a VOA (transmittance: $\eta$ ) can realize a PNR detector [22]. (b) An equivalent model to (a), which means the two models will produce the same output given the same input.

transmittances $\eta \in\left\{\eta_{0}, \eta_{1}, \eta_{2}\right\}$ are needed [22]. One can choose $\eta_{0}=1$,

$$
p\left(\eta_{0}=1\right)=(1-\lambda) p_{0}^{\prime}
$$

Then one has

$$
\begin{aligned}
& \frac{p\left(\eta_{1}\right)}{1-\lambda} \geq p_{0}^{\prime}+\left(1-\eta_{1}\right) p_{1}^{\prime} \\
& \frac{p\left(\eta_{1}\right)}{1-\lambda} \leq p_{0}^{\prime}+\left(1-\eta_{1}\right) p_{1}^{\prime}+\left(1-\eta_{1}\right)^{2}\left(1-p_{0}^{\prime}-p_{1}^{\prime}\right)
\end{aligned}
$$


from which one gets

$$
p_{1}^{\prime} \leq \frac{p\left(\eta_{1}\right)-p\left(\eta_{0}\right)}{(1-\lambda)\left(1-\eta_{1}\right)}=\overline{p_{1}^{\prime}}, p_{1}^{\prime} \geq \frac{p\left(\eta_{1}\right)-p\left(\eta_{0}\right)\left[1-\left(1-\eta_{1}\right)^{2}\right]-(1-\lambda)\left(1-\eta_{1}\right)^{2}}{(1-\lambda)\left[1-\eta_{1}-\left(1-\eta_{1}\right)^{2}\right]}=\underline{p_{1}^{\prime}} .
$$

In a similar way, one has

$$
\begin{aligned}
\frac{p\left(\eta_{2}\right)}{1-\lambda} & \geq p_{0}^{\prime}+\left(1-\eta_{2}\right) p_{1}^{\prime}+\left(1-\eta_{2}\right)^{2} p_{2}^{\prime} \geq p_{0}^{\prime}+\left(1-\eta_{2}\right) \underline{p_{1}^{\prime}}+\left(1-\eta_{2}\right)^{2} p_{2}^{\prime} \\
\frac{p\left(\eta_{2}\right)}{1-\lambda} & \leq p_{0}^{\prime}+\left(1-\eta_{2}\right) p_{1}^{\prime}+\left(1-\eta_{2}\right)^{2} p_{2}^{\prime}+\left(1-\eta_{2}\right)^{3}\left(p_{3}^{\prime}+p_{4}^{\prime}+p_{5}^{\prime}+\cdots\right) \\
& \leq\left[1-\left(1-\eta_{2}\right)^{3}\right] p_{0}^{\prime}+\left[1-\eta_{2}-\left(1-\eta_{2}\right)^{3}\right] \overline{p_{1}^{\prime}}+\left[\left(1-\eta_{2}\right)^{2}-\left(1-\eta_{2}\right)^{3}\right] p_{2}^{\prime}+\left(1-\eta_{2}\right)^{3},
\end{aligned}
$$

from which the upper and lower bounds for $p_{2}^{\prime}$ can be estimated as

$$
\begin{aligned}
& p_{2}^{\prime} \leq \frac{p\left(\eta_{2}\right)-p\left(\eta_{0}\right)-(1-\lambda)\left(1-\eta_{2}\right) \underline{p_{1}^{\prime}}}{(1-\lambda)\left(1-\eta_{2}\right)^{2}}=\overline{p_{2}^{\prime}} \\
& p_{2}^{\prime} \geq \frac{p\left(\eta_{2}\right)-\left[1-\left(1-\eta_{2}\right)^{3}\right] p\left(\eta_{0}\right)-(1-\lambda)\left[1-\eta_{2}-\left(1-\eta_{2}\right)^{3}\right] \overline{p_{1}^{\prime}}-(1-\lambda)\left(1-\eta_{2}\right)^{3}}{(1-\lambda)\left[\left(1-\eta_{2}\right)^{2}-\left(1-\eta_{2}\right)^{3}\right]}=\underline{p_{2}^{\prime}} .
\end{aligned}
$$

In conclusion, based on the recorded data $\left\{p\left(\eta_{0}\right), p\left(\eta_{1}\right)\right.$, $\left.p\left(\eta_{2}\right)\right\}$, Alice can estimated the parameters $\left\{a_{0}^{\prime}{ }^{L}, a_{0}^{U}, a_{1}^{\prime L}\right.$, $\left.a_{1}^{U}, a_{2}^{\prime}{ }^{L}, a_{2}^{U}\right\}$ as in Eqs. (12) and (14)-(16). The scheme in Fig. 4 can easily be realized with current technology. As for the effect of statistical fluctuation, one can use the randomsampling theory as before to consider the fluctuation of the $\left\{p\left(\eta_{0}\right), p\left(\eta_{1}\right), p\left(\eta_{2}\right)\right\}$ with a confidence level so that we still can bound $\left\{a_{0}^{\prime L}, a_{0}^{U}, a_{1}^{\prime L}, a_{1}^{U}, a_{2}^{\prime L}, a_{2}^{U}\right\}$.

\section{DISCUSSION AND CONCLUSION}

The results in Fig. 2] show that: (i) The performance of a QKD system with an untrusted source is close to that of a trusted source, when the source is monitored efficiently and the data size is large enough; (ii) finite-data size has negative effect on the secure key rate; (iii) the method in Ref. [15] is more sensitive to statistical fluctuation and needs a larger data size than the method proposed in this paper.

In the passive PNA scheme proposed in Ref. [15] (see Fig. 5 in Appendix A), Alice uses a PNA to monitor the probability of the untagged bits in the untrusted source, after which, one can estimate the lower bound of the secure key rate with a confidence level as shown in Eq. (A1). When the confidence level is set to be constant (e.g., $1-10^{-6}$ ), the estimation resolution $\varepsilon$ for the probability of untagged bits is only decided by the data size $N$ (ignoring the effect of detection noise), where the confidence level is $1-2 \exp \left(-N \varepsilon^{2} / 4\right)[15]$. However, the secure key rate in Ref. [15] is sensitive to the estimation resolution $\varepsilon$, and will reduce greatly when $\varepsilon$ increases (see Fig. 6 in Appendix A). When the data size $N$ decreases, the resolution $\varepsilon$ has to increase to keep the constant confidence level, and, thus, the key rate will reduce.

While in the scheme shown in Fig. 1, Alice uses a PNR detector to monitor the probability of vacuum, one-photon, and two-photon states for the signal and decoy sources, respectively. Because of the low intensity of the output pulses at P4 (e.g., $\mu_{s}=0.5, \mu_{d}=0.1$ ), the vacuum, one-photon, and twophoton pulses are dominant in pulses, and Alice can gain most of the information about the statistics of the untrusted source at P4 based on the recorded data of the PNR detector. In our scheme, six parameters are monitored, and more information is gained than from the scheme in Ref. [15]. Mathematically, the formulas shown by Eq. (4) are not so sensitive to the estimation resolution of $\left\{a_{0}^{\prime}{ }^{L}, a_{0}^{U}, a_{1}^{\prime}{ }^{L}, a_{1}^{U}, a_{2}^{\prime}{ }^{L}, a_{2}^{U}\right\}$ compared to that in Ref. [15] so that it does not require a very large data size to work efficiently, as shown in Fig. 2(a). When the data size is $N \geq 10^{8}$, the performance of the scheme is very close to that of the trusted source. In the asymptotic case where Alice sends infinitely long bits of sequence $(N \sim \infty)$, the performance will be the same as that of a trusted source as shown in Appendix B.

The results in Fig. 3 show that: (i) Given a PNR detector with the same dark count rate, the performance of a system with an untrusted source will be better when the data size increases; (ii) given the same data size, the performance of a system with an untrusted source will reduce when the dark count rate increases. The performance of the scheme in Fig. 1 is quite sensitive to the detection noise of the PNR detector. It is shown that when the data size is $N \geq 10^{8}$ and the dark count rate of the PNR detector is $\lambda \leq 0.5$ counts/pulse, which are realizable by current techniques [30-33], this scheme can still work efficiently.

In conclusion, we propose an experimental scheme to verify 
the key parameters needed in Refs. [19, 20]. The practical issues due to detection noise and finite-data-size fluctuation are analyzed. We also propose a realization of the PNR detector based on the detector-decoy method, which is very practical in real experiment. Therefore, the passive scheme with a PNR detector is highly practical to solve the untrusted source problem in the two-way plug-and-play QKD system. This passive scheme is also applicable to monitor the intensity fluctuation in a one-way QKD system, where an active scheme has been proposed and has been tested experimentally [26].

We remark that the effect of parameter fluctuations has not yet been included in the security analysis. The effective method to deal with the parameter fluctuations [19] is encouraged to be applied in the passive scheme.

\section{Acknowledgments}

This work was supported by the Key Project of National Natural Science Foundation of China (Grant No. 60837004) and the National Hi-Tech Research and Development (863) Program. X. Peng acknowledges financial support from the China Postdoctoral Science Foundation (Grant No. 20100470134).

\section{Appendix A: Passive scheme method in [15]}

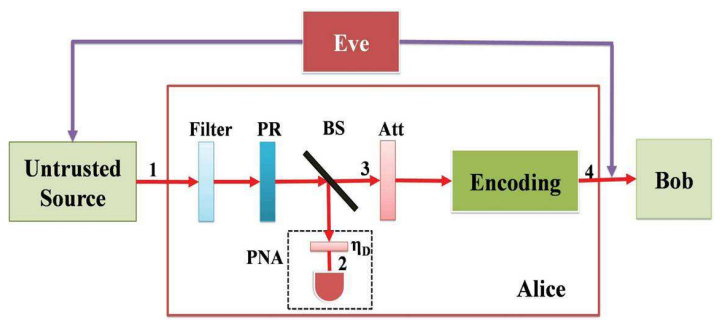

FIG. 5: (Color online) The model of the passive PNA scheme in Ref. [14-16]. The untrusted source prepared by Eve passes through an optical filter and a PR. Then, a BS with transmittance $\eta_{B S}$ separates it into two beams: One goes to a PNA with efficiency $\eta_{D}$, and the other is attenuated by an Att with efficiency $\eta_{s(d)}$ for the signal (decoy) state and is encoded before being from sent out of Alice's side.

The passive PNA scheme in Ref. [15] is shown in Fig. [5, For simplicity, one can set $\eta_{D}\left(1-\eta_{B S}\right)=\eta_{B S}$ so that the PND at position 2 is the same as that at position 3 in Fig. 5. Define the pulses, whose photon number is $M \in\left[M_{\min }, M_{\max }\right]$ at position 3, as untagged bits. A BS and a PNA are used to record the frequency of untagged bits experimentally.

Suppose that $N$ pulses are sent from Alice to Bob. Alice and Bob do not know which bits are untagged bits. Let $N_{\text {untagged }}$ denote the number of detected pulses by the PNA when the recorded photoelectron number at position 2 belongs to $\left[M_{\min }, M_{\max }\right]$, and $\Delta=N_{\text {untagged }} / N$. From the recorded data in the PNA, one can estimate that at least $(1-\Delta-\varepsilon) N$ pulses

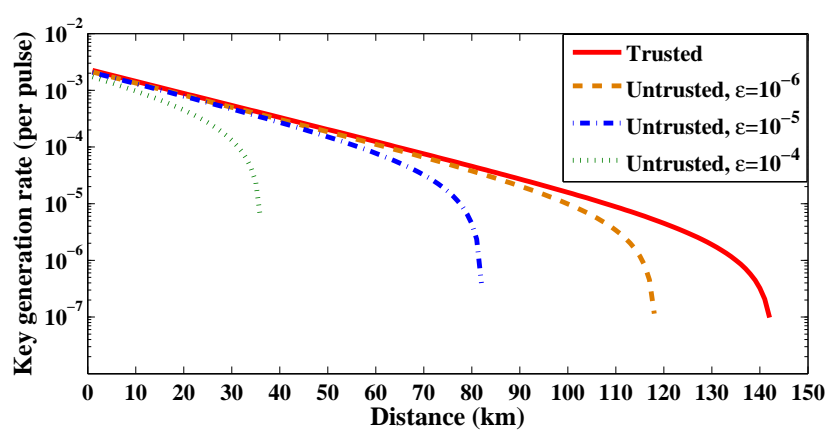

FIG. 6: (Color online) Simulation result of the three-intensity decoy state protocol for the trusted source and the untrusted source with different estimation resolutions $\varepsilon=10^{-6}, 10^{-5}, 10^{-4}$, respectively. Based on the passive PNA scheme in Fig. 5, a BS and a noiseless PNA are used to verify the lower bound of the probability of untagged bits. Experimental parameters are cited from Table【

are untagged bits with a confidence $1-2 \exp \left(-N \varepsilon^{2} / 4\right)$ where $\varepsilon$ is a small positive parameter [15].

Alice can measure the overall gain $Q_{s(d)}$ and the QBER $E_{s(d)}$ for signal (decoy) pulses, respectively, while she does not know the gain and the QBER for the untagged bits. The upper and lower bounds of the gain of the untagged bits for the signal (decoy) source can be estimated as

$$
\overline{Q_{s(d)}}=\frac{Q_{s(d)}}{1-\Delta-\varepsilon}, \underline{Q_{s(d)}}=\max \left\{0, \frac{Q_{s(d)}-\Delta-\varepsilon}{1-\Delta-\varepsilon}\right\} .
$$

The upper and lower bounds for the QBER of the untagged bits can be estimated as

$$
\overline{Q_{s} E_{s}}=\frac{Q_{s} E_{s}}{1-\Delta-\varepsilon}, \underline{Q_{s} E_{s}}=\max \left\{0, \frac{Q_{s} E_{s}-\Delta-\varepsilon}{1-\Delta-\varepsilon}\right\},
$$

for signal states, and

$$
\overline{Q_{d} E_{d}}=\frac{Q_{d} E_{d}}{1-\Delta-\varepsilon}, \underline{Q_{d} E_{d}}=\max \left\{0, \frac{Q_{d} E_{d}-\Delta-\varepsilon}{1-\Delta-\varepsilon}\right\},
$$

for decoy states. For the untagged bits, one can show that the upper and lower bounds of the probability that the output photon number at position 4 is $n$ for signal (decoy) pulses are

$$
\begin{aligned}
& \overline{P_{n}^{s(d)}}= \begin{cases}\left.\left(1-\eta_{s(d)}\right)\right)_{\min } & n=0, \\
\left(\begin{array}{c}
M_{\max } \\
n
\end{array}\right) \eta_{s(d)}^{n}\left(1-\eta_{s(d)}\right)^{M_{\max }-n} & 1 \leq n \leq M_{\max }, \\
0 & n>M_{\max },\end{cases} \\
& \underline{P_{n}^{s(d)}}= \begin{cases}\left(1-\eta_{s(d)}\right)^{M_{\max }} & n=0, \\
\left(\begin{array}{c}
M_{\min } \\
n
\end{array}\right) \eta_{s(d)}^{n}\left(1-\eta_{s(d)}\right)^{M_{\min }-n} & 1 \leq n \leq M_{\min }, \\
0 & n>M_{\min },\end{cases}
\end{aligned}
$$

under the condition $M_{\max } \eta_{s(d)}<1$. 
When the lower bounds of the probability of the untagged bits are known by Alice, the secure key rate for the threeintensity decoy-state protocol with an untrusted source is [13]

$$
R=\frac{1}{2}\left\{-Q_{s} H_{2}\left(E_{s}\right)+(1-\Delta-\varepsilon) \underline{Q_{1}^{s}}\left[1-H_{2}\left(\overline{e_{1}^{s}}\right)\right]\right\},
$$

where

$$
\begin{aligned}
\underline{Q_{1}^{s}}= & \frac{\frac{P_{1}^{s}}{\overline{P_{1}^{d}}} \underline{P 2}_{2}^{s}-\underline{P_{1}^{s}} \overline{P_{2}^{d}}}{} \times\left\{\underline{Q_{d} P_{2}^{s}}-\overline{Q_{s}} \overline{P_{2}^{d}}+\underline{P_{0}^{s}} \overline{P_{2}^{d}} Q_{0}-\right. \\
& \left.\overline{P_{0}^{d}} \underline{P_{2}^{s}} Q_{0}-\frac{\left(M_{\max }-M_{\min }\right)\left(1-\eta_{d}\right)^{M_{\max }-M_{\min }-1} \underline{P_{2}^{s}}}{\left[M_{\min }+1\right] !}\right\},
\end{aligned}
$$

and

$$
\overline{e_{1}^{s}}=\frac{\overline{E_{s} Q_{s}}-\underline{P_{0}^{s}} \underline{E_{0} Q_{0}}}{\underline{Q_{1}^{s}}} .
$$

For testing the effects of $\varepsilon$ onto the secure key rate, we choose an untrusted source of Poissonian statistics to perform the simulations based on the three-intensity decoy-state protocol. Suppose that the untrusted source has Poissonian PND with an APN of $7.69 \times 10^{6}$ at position 1 of Fig. 5. Set $\eta_{s}=5 \times 10^{-7}$, and $\eta_{d}=1 \times 10^{-7}$. The other experimental parameters are chosen to be the same as in Table I. The values of $M_{\max }$ and $M_{\text {min }}$ are chosen to be constant. The results in Fig. 6 show that the final key rate is very sensitive to the value of $\varepsilon$.

Suppose that Alice has a noiseless PNA, and the estimation confidence level is set to be constant

$$
1-2 e^{-N \varepsilon^{2} / 4}=1-10^{-6} .
$$

The estimation resolution $\varepsilon$ is only decided by the data size $N$. When the data size $N$ decreases, the resolution $\varepsilon$ has to increase to keep the constant confidence level, and, thus, the key rate will reduce greatly as shown in Fig. 2(b).

\section{Appendix B: Asymptotic case of method in [19]}

In the asymptotic case, Alice sends the infinitely long bits sequence $(N \sim \infty)$. Therefore, one can consider $\varepsilon, \varepsilon^{\prime} \sim 0$ in Eqs. (7), (9), or (11) while still having the confidence level 1. Suppose that the PND of the untrusted source is Poissonian with an APN of $\mu_{s(d)}$ for the signal (decoy) source at P4 in Fig 1 One has

$$
\begin{aligned}
& a_{0}^{U}=e^{-\mu_{d}}, a_{1}^{U}=\mu_{d} e^{-\mu_{d}}, a_{2}^{U}=\frac{\mu_{d}^{2}}{2} e^{-\mu_{d}} \\
& a_{0}^{\prime L}=e^{-\mu_{s}}, a_{1}^{\prime L}=\mu_{s} e^{-\mu_{s}}, a_{2}^{\prime L}=\frac{\mu_{s}^{2}}{2} e^{-\mu_{s}}
\end{aligned}
$$

Then, one can estimate

$$
\begin{aligned}
Q_{1}^{s}=Q_{s} \Delta_{1}^{s} & \geq \frac{a_{1}^{\prime L}\left(a_{2}^{\prime L} Q_{d}-a_{2}^{U} Q_{s}-a_{2}^{\prime}{ }^{L} a_{0}^{U} Q_{0}+a_{2}^{U} a_{0}^{{ }^{L}} Q_{0}\right)}{a_{1}^{U} a_{2}^{\prime L}-a_{1}^{\prime}{ }^{L} a_{2}^{U}} \\
& =\frac{\mu_{s}}{\mu_{s}^{2}-\mu_{s} \mu_{d}}\left(Q_{d} e^{-\mu_{d}}-Q_{s} e^{-\mu_{s}} \frac{\mu_{d}^{2}}{\mu_{s}^{2}}-\frac{\mu_{s}^{2}-\mu_{d}^{2}}{\mu_{s}^{2}} Q_{0}\right),
\end{aligned}
$$

which is exactly the same as the case for a trusted source.
[1] C. H. Bennett and G. Brassard, in Proceedings of the IEEE International Conference on Computers, Systems, and Signal Processing (IEEE, New York, 1984), p. 175.

[2] H. Inamori, N. Lütkenhaus, and D. Mayers, Eur. Phys. J. D 41, 599 (2007).

[3] D. Gottesman, H. K. Lo, N. Lütkenhaus, and J. Preskill, Quantum Inf. Comput. 4, 325 (2004).

[4] W. Y. Hwang, Phys. Rev. Lett. 91, 057901 (2003).

[5] H. K. Lo, Proceeding of 2004 IEEE Int. Symp. on Inf. Theor., Chicago (IEEE, New York, 2004), p17.

[6] X. B. Wang, Phys. Rev. Lett. 94, 230503 (2005).

[7] H. K. Lo, X. Ma, and K. Chen, Phys. Rev. Lett. 94, 230504 (2005).

[8] X. B. Wang, Phys. Rev. A 72, 012322 (2005).

[9] X. Ma, B. Qi, Y. Zhao, and H. K. Lo, Phys. Rev. A 72, 012326 (2005).

[10] W. Mauerer and C. Silberhorn, Phys. Rev. A 75, 050305(R) (2007).

[11] X. B. Wang, T. Hiroshima, A. Tomita, and M. Hayashi, Phys. Rep. 448, 1 (2007).

[12] N. Gisin, S. Fasel, B. Kraus, H. Zbinden, and G. Ribordy, Phys. Rev. A 73, 022320 (2006).

[13] Y. Zhao, B. Qi, and H. K. Lo, Phys. Rev. A 77, 052327 (2008).
[14] X. Peng, H. Jiang, B. Xu, X. Ma, and H. Guo, Opt. Lett. 33, 2077 (2008).

[15] Y. Zhao, B. Qi, H. K. Lo, and L. Qian, New J. Phys. 12, 023024 (2010).

[16] X. Peng, B. Xu, and H. Guo, Phys. Rev. A 81, 042320 (2010).

[17] X. B. Wang, Phys. Rev. A 75, 052301 (2007).

[18] X. B. Wang, C. Z. Peng, and J. W. Pan, Appl. Phys. Lett. 90, 031110 (2007).

[19] X. B. Wang, C. Z. Peng, J. Zhang, L. Yang and J. W. Pan, Phys. Rev. A 77, 042311 (2008).

[20] X. B. Wang, L. Yang, C. Z. Peng, and J. W. Pan, New J. Phys. 11, 075006 (2009).

[21] J. Z. Hu and X. B. Wang, arXiv: quant-ph/1004.3730 (2010).

[22] T. Moroder, M. Curty, and N. Lütkenhaus, New J. Phys. 11, 045008 (2009).

[23] M. Curty, T. Moroder, X. Ma, and Lütkenhaus, Opt. Lett. 34, 3238 (2009).

[24] M. Curty, X. Ma, B. Qi, and T. Moroder, Phys. Rev. A 81, 022310 (2010).

[25] Y. Adachi, T. Yamamoto, M. Koashi, and N. Imoto, New J. Phys. 11, 113033 (2009).

[26] F. X. Xu, Y. Zhang, Z. Zhou, W. Chen, Z. F. Han, and G. C. Guo, Phys. Rev. A 80, 062309 (2009). 
[27] M. O. Scully and M. S. Zubairy, Quantum Optics (Cambridge University Press, Cambridge U.K., 1997).

[28] J. V. Uspensky, Introduction to Mathematical Probability (McGraw-Hill, New York, 1937).

[29] C. Gobby, Z. L. Yuan, and A. J. Shields, Appl. Phys. Lett. 84, 3762 (2004).

[30] D. Achilles, C. Silberhorn, C. Sliwa, K. Banaszek, and I. A. Walmsley, Opt. Lett. 28, 2387 (2003).

[31] D. Achilles, C. Silberhorn, C. Sliwa, K. Banaszek, I. A. Walm- sley, M. J. Fitch, B. C. Jacobs, T. B. Pittman, and J. D. Franson, J. Mod. Opt. 51, 1499 (2004).

[32] D. Rosenberg, A. E. Lita, A. J. Miller,and S. W. Nam, Phys. Rev. A 71, 061803 (2005).

[33] G. Zambra, A. Andreoni, M. Bondani, M. Gramegna, M. Genovese, G. Brida, A. Rossi, and M. G. A. Paris, Phys. Rev. Lett. 95, 063602 (2005). 